Bull. Austral. Math. Soc.

Vol. 45 (1992) [1-7]

\title{
ABELIAN GROUPS THAT ARE UNIONS OF PROPER SUBGROUPS
}

\author{
K.P.S. BHASKara RAO AND J.D. REID
}

The abelian groups that can be written as a union of proper subgroups are characterised, all the ways that this can be done for a given group are indicated, and the minimal number of subgroups necessary for such a decomposition of a given group is determined.

\section{INTRODUCTION}

It is an elementary fact that no group is the union of two proper subgroups. On the other hand, a finite non-cyclic group is the union of its (finitely many) cyclic subgroups, all of which are proper. One can consider the related question of whether a vector space over a field can be written as a finite union of proper subspaces. Some results on this, and further references, may be found in [2]. The fact that finite dimensional spaces over fields of characterictic 0 (or over any infinite field) cannot be so written is the basis of an elegant proof of the theorem of the primitive element in field theory (see for example [3], Corollary to Theorem 1, p.34). More general questions concern the possibility of representing vector spaces as unions of hyperplanes.

In this note we characterise the abelian groups that can be written as a finite union of proper subgroups; we determine all the ways in which this can be done for a given group; and we determine the minimal number of subgroups necessary for such a decomposition of a given group. All groups under discussion are abelian. See [1] for a general reference.

\section{NeUMANN'S LEMma}

Our approach is based on a generalisation of the following result of Neumann (see $[1$, p.31]). (We are indebted to K.M. Rangaswamy for this reference):

Lemma. Let $S_{1}, \ldots, S_{n}$ be subgroups of the group $A$ such that $A$ is the settheoretic union of finitely many cosets

$$
A=\left(a_{1}+S_{1}\right) \cup \ldots \cup\left(a_{n}+S_{n}\right), a_{i} \in A .
$$

Then some one of $S_{1}, \ldots, S_{n}$ is of finite index in $A$.

This lemma can be generalised to

Received 4 January 1991

Copyright Clearance Centre, Inc. Serial-fee code: 0004-9729/92 \$A2.00+0.00. 
ThEOREM 1. Let $S_{1}, \ldots, S_{n}$ be subgroups of the group $A$ such that $A$ is the set-theoretic union of finitely many cosets

$$
A=\left(a_{1}+S_{1}\right) \cup \ldots \cup\left(a_{n}+S_{n}\right)
$$

$a_{i} \in A$, and with the further property that no proper subunion equals $A$. Then each of $S_{1}, \ldots, S_{n}$ has finite index in $A$.

Proof: By the Lemma, one of the $S_{i}$, say $S_{1}$, has finite index in $A$. Suppose that in fact $S_{1}, \ldots, S_{k}$ have finite index in $A$. We show that, if $k<n$, then at least one more $S_{j}, k<j \leqslant n$ has finite index in $A$.

Thus, let $S=S_{1} \cap \ldots \cap S_{k}$. Then $S$ is also of finite index in $A$ so each of the cosets $a_{i}+S_{i}, 1 \leqslant i \leqslant k$, is a finite union of cosets of $S$. Since $\left(a_{1}+S_{1}\right) \cup \ldots \cup\left(a_{k}+S_{k}\right)$ is not equal to $A$, some coset $x+S$ is disjoint from this union $\left(a_{1}+S_{1}\right) \cup \ldots \cup\left(a_{k}+S_{k}\right)$. Hence

$$
x+S \subseteq\left(a_{k+1}+S_{k+1}\right) \cup \ldots \cup\left(a_{n}+S_{n}\right)
$$

so $S$ itself is contained in $\bigcup_{t=k+1}^{n}\left(a_{t}-x+S_{t}\right)$. Thus any coset $y+S$ is contained in $\bigcup_{t=k+1}^{n}\left(a_{t}-x+y+S_{t}\right)$ and it follows that $a_{i}+S_{i}, 1 \leqslant i \leqslant k$, being a union of finitely many such cosets, is contained in the union of finitely many cosets of the subgroups $S_{k+1}, \ldots, S_{n}$. Therefore $A$ is contained in the union of finitely many such cosets and by Neumann's Lemma, some one of $S_{k+1}, \ldots, S_{n}$ is of finite index in $A$. This completes the proof.

Corollary 1.1 . Let $S_{1}, \ldots, S_{n}$ be proper subgroups of $A$ such that

$$
A=S_{1} \cup \ldots \cup S_{n}
$$

and no proper subunion equals $A$. Put $S=S_{1} \cap \ldots \cap S_{n}$. Then $A / S$ is a finite group.

\section{MAIN RESULTS}

To facilitate the statement of the main result we make the following

Definition: A finite collection of proper subgroups $S_{i}, 1 \leqslant i \leqslant n$, of $A$ is called a decomposition of $A$ if $A$ is the union of the $S_{i}$ and no proper subunion of the $S_{i}$ equals $A$ (equivalently, no $S_{i}$ is contained in the union of the others).

DEFinition: Let $B$ be a homomorphic image of the group $A$ under the homomorphism $\varphi$. Suppose that $B_{1}, \ldots, B_{n}$ is a decomposition of $A$. Then $A=\bigcup_{i=1}^{n} \varphi^{-1}\left(B_{i}\right)$ will be called the decomposition induced by that of $B$ via $\varphi$. 
Theorem 2. Let $A$ be an abelian group. Then $A$ can be written as the union of a finite number of proper subgroups if and only if $A$ has a finite non-cyclic homomorphic image. Every decomposition of $A$ into a union of finitely many proper subgroups is induced by a decomposition of some finite homomorphic image into proper subgroups.

Proof: Let $A$ be the union of a finite collection of proper subgroups $S_{i}, 1 \leqslant i \leqslant$ $n$. Without loss of generality we may assume that the $S_{i}$ form a decomposition of $A$, that is that no $S_{i}$ is contained in the union of the others. Denote by $S$ the intersection of the $S_{i}, 1 \leqslant i \leqslant n$. Then the group $B=A / S$ is finite by the corollary above, and $B$ is the union of its subgroups $B_{i}=S_{i} / S$, which are clearly proper subgroups of $B$. Then $B$ is not cyclic, otherwise a generator would belong to some $B_{i}$, which would then be all of $B$. Clearly the given decomposition of $A$ coincides with that induced by the decomposition of $B$ into the union of the $B_{i}$.

Conversely if $A$ has a finite non-cyclic homomorphic image $B$ under the homomorphism $\varphi$, say, then $B$ is the union of its cyclic subgroups, which are finite in number and certainly all proper. Then $A$ has a decomposition, namely the one induced via $\varphi$ by that of $B$.

Corollary 2.1. In any decomposition of an abelian group $A$ into a union of finitely many proper subgroups $S_{i}$ the maximal divisible subgroup, $D$, of $A$ is contained in each of the $S_{i}$. Every decomposition of $A$ is induced by a decomposition of the reduced quotient $A / D$ of $A$. In particular a divisible abelian group admits no decomposition into a union of finitely many proper subgroups.

In view of this corollary, the decomposition theory (in our sense) of a group $A$ is determined by that of its reduced part.

Definition: For an abelian group $G$ we denote by $k(G)$ the smallest number of proper subgroups of $G$ whose union is $G$ if such a union exists; $k(G)=\infty$ otherwise.

Here, " $\infty$ " is to be interpreted as denoting an object that exceeds every natural number. We will see later that any (non-cyclic) abelian group can be written as the union of countably many proper subgroups, so that once this is known, $\infty$ could be taken to be $\aleph_{0}$.

Observe that, if $H$ is a homomorphic image of $G$, then $k(G) \leqslant k(H)$ since any decomposition of $H$ induces one of $G$ with the same number of subgroups. We have the following

Theorem 3. Suppose that the finite group $G$ is the direct sum of $H$ and $K$ and that $H$ and $K$ have relatively prime orders. Then

$$
k(G)=\operatorname{Min}[k(H), k(K)]
$$

Proof: Let $G=H \oplus K$, with $H$ of order $n, K$ of order $m$ and $\operatorname{gcd}(n, m)=1$. 
Then $H$ and $K$ are homomorphic images of $G$ so that $k(G) \leqslant k(H)$ and $k(G) \leqslant k(K)$; hence $k(G) \leqslant \operatorname{Min}[k(H), k(K)]$.

Suppose that $G=S_{1} \cup \ldots \cup S_{k}$, with $k=k(G)$ least. Expanding each $S_{i}$ to a maximal subgroup if necessary, we may assume that each $S_{i}$ is maximal in $G$, so has prime index $p_{i}$, say. Then $p_{i}$ divides $n$ or $m$ but not both. Now $n G=K$ and $m G=H$ so if $p_{i}$ divides $n$ then $K=n G \subseteq p_{i} G \subseteq S_{i}$, and if $p_{i}$ divides $m$ then $H \subseteq S_{i}$ by a similar argument. Thus, for each $i$, either $H \subseteq S_{i}$ or $K \subseteq S_{i}$ (but not both since $S_{i}$ is a proper subgroup of $G$ ). We assume the numbering so chosen that $S_{1}, \ldots, S_{t}$ contain $H$, the remaining $S_{j}$ contain $K$.

Now if there were an element $x$ in $K$ but not in any of the $S_{i} 1 \leqslant i \leqslant t$, and an element $y$ in $H$ but not in any of the remaining $S_{j}, t+1 \leqslant j \leqslant k$, then consider the element $x+y$ of $G$. It must lie in some $S_{i}$. If $i \leqslant t$ then both $y$ and $x+y$ lie in $H$, so $x$ is also in $H$. But then $x$ lies in $H \cap K=0$, a contradiction. Similarly, $x+y$ cannot lie in $S_{j}$ for $j>t$. Thus, either $K=S_{1} \cap K \cup \ldots \cup S_{t} \cap K$, in which case $k(G) \geqslant t \geqslant k(K)$, or $H=\bigcup_{j=t+1}^{k} S_{j} \cap H$ so $k(G) \geqslant k-t \geqslant k(H)$. In either case $k(G) \geqslant \operatorname{Min}[k(H), k(K)]$ as required.

COROLlaRY 3.1. Let $G$ be a finite group with primary components

$$
G_{p}=\left\{x \in G: p^{m} x=0 \text { for some integer } m\right\} .
$$

Then $k(G)=\operatorname{Min}\left\{k\left(G_{p}\right): p\right.$ prime $\}$.

We can now determine the value of $k(G)$ for any $G$.

THEOREM 4. Let $G$ be an abelian group which can be written as a union of finitely many proper subgroups. Then $k(G)$ equals $p+1$ where $p$ is the smallest prime for which $G / p G$ is not cyclic (that is, for which $G / p G$ has dimension greater than one over the field $Z / p Z$ ).

Proof: By Theorem 2 it suffices to consider finite groups. Then Corollary 3.1 reduces the problem to the case of primary groups, that is, groups in which every element has order a power of a fixed prime, say $p$. Write such a group $G$ as the union of $S_{i}, 1 \leqslant i \leqslant k$, with $k$ minimal. We may expand each $S_{i}$ to a maximal subgroup of $G$ and the new groups still have union $G$. Thus we may assume our original $S_{i}$ are maximal subgroups of $G$, so each has prime index in $G$. Since $G$ is a p-group, this means that $p G \subseteq S_{i}$ for all $i$. Hence the $S_{i}$ induce a decomposition of $G / p G$ into a union of $k$ proper subgroups. But $G / p G$ is a vector space over the field $Z / p Z$, and by Theorem 1 of $[2]$ we have $k \geqslant p+1$, as required.

\section{GROUPS WITH NO DECOMPOSITION}

One can ask for a determination of those groups that have no decomposition into a 
union of proper subgroups. Of course these are the groups that have no finite non-cyclic homomorphic images, but we give here in a sense a description of them all. We need a small amount of notation.

For an abelian group $G$ denote by $T$ the torsion subgroup of $G$, that is, the subgroup of elements of finite order in $G$. Then $G / T$ is a torsion free group that we denote by $W$. By the $p$-rank of a group $A$ we understand the dimension of $A / p A$ as a vector space over the field $Z / p Z$. Here of course $p$ represents a prime. We write $r_{p}(A)$ for the $p$-rank of $A$.

THEOREM 5. For an abelian group $G$, the following are equivalent:

(a) $G$ has no finite non-cyclic homomorphic images;

(b) $r_{p}(T)+r_{p}(W) \leqslant 1$ for all $p$.

Proof: Suppose that $G$ has no finite non-cyclic homomorphic images and let $p$ be a prime. Then $G / p G$ is cyclic and since $G / p G \cong T / p T \oplus W / p W$ it is clear that condition (b) holds.

Conversely, suppose that $r_{p}(T)+r_{p}(W) \leqslant 1$ for all $p$. Since any finite homomorphic image of $G$ is itself an image of a group of the form $G / n G$ for some integer $n$, and since $G / n G$ is isomorphic to the product of the groups $G / p^{e} G$ where $n=\Pi p^{e}$, it suffices to show that the groups $G / p^{e} G$ are cyclic for all primes $p$ and non-negative integers $e$.

Observe that for the torsion free group $W, W / p^{e} W$ is cyclic since its socle, that is, $\{x: p x=0\}$ is $p^{e-1} W / p^{e} W$ which is isomorphic to $W / p W$ and so is (0 or) cyclic. Similarly, $T / p^{e} T$ is cyclic under our hypotheses. We consider two cases:

(1) $r_{p}(T)=0$ so that $p T=T$. Then $T \subseteq p^{e} G$ so $G / p^{e} G \cong W / p^{e} W$ and is cyclic by the remarks above.

(2) $r_{p}(W)=0$ so that $p W=W$. Here $p^{e} G+T=G$ and it follows that

$$
G / p^{e} G=\left(p^{e} G+T\right) / p^{e} G \cong T /\left(p^{e} G \cap T\right)=T p^{e} T,
$$

hence is again cyclic (or 0 ).

Since one of these cases must occur this proves the theorem.

Corollary 5.1. If $G$ has no decomposition into a union of proper subgroups, then its torsion subgroup, $T$, has the form $T=\bigoplus_{p} Z\left(p^{k_{p}}\right), 0 \leqslant k_{p} \leqslant \infty$, where $Z\left(p^{k_{p}}\right)$ is cyclic of order $p^{k_{p}}$ if $k<\infty, Z\left(p^{\infty}\right)$ otherwise.

There are several well known classes of groups that can serve as examples of the exceptional groups discussed in the last theorem. The groups in the first two of these do not split into the direct sum of torsion and torsion free parts, and so perhaps indicate 
the complexity of structure of the groups under discussion. Some details on these groups may be found in [1, vol II, p.186].

(i) Let $T$ be the direct sum of cyclic p-groups for a set of distinct primes p. Form $G=\operatorname{Ext}(Q / Z, T)$. Then $T$ can be identified with the torsion subgroup of $G$ and the corresponding quotient group $W$ is divisible. If $T$ is not finite $G$ is a simple example of an adjusted cotorsion group.

(ii) Let $T$ be a direct sum of cyclic groups $C(p)$ of order $p$ for some set of distinct primes $p$ and form the product

$$
G=\Pi C(p)
$$

Then $T$ is again the torsion subgroup of $G$ and the quotient $W$ is divisible.

(iii) The so-called Murley groups are exactly the torsion free groups (of finite rank) of $p$-rank at most 1 for each prime $p$, and hence are among the groups under discussion.

We have seen that the torsion subgroup $T$ of a group $G$ that has no decomposition into a (finite) union of proper subgroups is rather special, in particular is countable, while the torsion free quotient $W=G / T$ can be structurally complicated. However, since $W / n W$ is cyclic for all positive integers $n$ and $W$ can be embedded in $\Pi_{n} W / n W$ if $W$ is reduced, it follows that such a group has cardinality at most equal to $c$. This upper bound is realised, for example, by the group $\Pi_{p} R_{p}$, where $R_{p}$ is the subgroup of the rationals $Q$ consisting of all rational numbers whose denominators are prime to p. It is clear that every finite image of this group is cyclic. Of course non-reduced groups satisfying our condition can be arbitrarily large since their divisible subgroups are unrestricted.

In contrast to the results we have obtained on the decomposability of groups into finite unions of proper subgroups we note that every noncyclic abelian group $G$ can be written as the union of countably many proper subgroups.

Indeed, we note first that if the group $G$ is itself countable (but noncyclic) we write it as the union of its countably many cyclic subgroups, all being of course proper. Otherwise, $G$ has a countable non-cyclic homomorphic image $H$ and $H$ can be written as a countable union of proper subgroups as we have noted. Then this yields, in the usual way, a representation of $G$ as a countable union of proper subgroups.

\section{REFERENCES}

[1] L. Fuchs, Abelian groups, Vol. I, II (Academic Press, New York, 1970).

[2] K.P.S. Bhaskara Rao and A. Ramachandra Rao, 'Unions and common complements of subspaces', Amer. Math. Monthly (to appear). 
[3] Pierre Samuel, Algebraic theory of numbers (Hermann, Paris, 1970).

Indian Statistical Institute

8th Mile, Mysore Road

Bangalore 560059

India
Department of Mathematics

Wesleyan University

Middletown, CT 06457

United States of America 